\title{
An Enigmatic Liver Mass in a Child
}

\author{
Ravi Thanage ${ }^{1}$, Shubham Jain ${ }^{2}$, Nikhil Sonthalia ${ }^{3}$, Suhas Udgirkar ${ }^{4}$, Sanjay Chandnani ${ }^{5}$, Qais Contractor ${ }^{6}$, Pravin Rathi ${ }^{7}$
}

\begin{abstract}
Hepatic space occupying lesions in childhood are a diagnostic challenge, as they are caused by a variety of malignant and nonmalignant disorders with a different prognosis and, of course, treatment. They are often misdiagnosed or diagnosed only after surgical resection. A 14-year-old boy presented with abdominal pain, evening-rise fever with loss of appetite and weight. The patient also developed jaundice after 2 months of above symptoms. Ultrasound of the abdomen showed an irregular infiltrative mass in segment IV of the liver. Gadobenate disodium magnetic resonance imaging done showed T1 hypointense and T2 hyperintense lesions in segment VIII of the liver with extension into porta with delayed enhancement suggestive of fibrous tumor. Liver biopsy showed extensive liver parenchymal fibrosis with a mixed inflammatory infiltrate with eosinophils. Bacterial, tubercular, and fungal culture of liver biopsy were negative. Although serum lgG4 levels were $7.88 \mathrm{~g} / \mathrm{L}(\mathrm{N}=1.9 \mathrm{~g} / \mathrm{L})$, lgG4 staining of liver biopsy was negative. The patient was started on prednisolone $1 \mathrm{mg} / \mathrm{kg}$ considering the diagnosis of inflammatory pseudotumor (IPT). Twenty days after starting the steroid, mass lesions were converted into multicystic abscess requiring antibiotics and pigtail drainage. On follow-up, patient had improved symptoms with mass lesions turned into small-sized abscess cavity. Hepatic IPTs are difficult to differentiate from malignant tumors, as they are rare and can have variable imaging findings. To avoid inadvertent surgery, histological confirmation of the hepatic mass is essential. Steroids should be used with caution with close follow-up to prevent iatrogenic complications, such as a chronic liver abscess. Keywords: Corticosteroids, Inflammatory pseudotumor, Liver abscess.

Euroasian Journal of Hepato-Gastroenterology (2019): 10.5005/jp-journals-10018-1307
\end{abstract}

\section{INTRODUCTION}

Hepatic space occupying lesions in childhood are a diagnostic challenge, as they are caused by a variety of malignant and nonmalignant disorders with a different prognosis and, of course treatment.

Despite recent advances in diagnostic imaging, sometimes it is difficult to differentiate between benign and malignant entities and most children should undergo a liver biopsy. Many children receive antitubercular therapy (ATT) or undergo surgery for possible malignant tumors without confirming diagnosis. We describe a case of a child with large apparent malignant hepatic space occupying lesion, initially treated as tubercular mass, turned out to be rare benign tumor that was managed conservatively.

\section{Case Description}

A 14-year-old boy was referred to our center for right upper abdominal pain and fever for 4 months and jaundice for 2 months. There was no history of abdominal distension, hematemesis, melena, cough with expectoration, burning micturition, or diarrhea. The patient was pale, icteric, and had nontender hepatomegaly $5 \mathrm{~cm}$ below the costal margin. The spleen was palpable $3 \mathrm{~cm}$ below the left costal margin. There were no ascites.

The patient was previously evaluated elsewhere for these complaints. As shown in Table 1, the patient had hyperbilirubinemia, raised levels of alkaline phosphatase and $\gamma$-glutamyltransferase (GGT). The etiology could not be ascertained as serological tests for hepatitis B and C, echinococcus, and brucellosis were negative. Tumor markers (a-fetoprotein, carcinoembryonic antigen (CEA), carbohydrate antigen 19.9 (CA-19. 9)) were normal. Upper gastrointestinal endoscopy showed small esophageal varices.

Abdominal ultrasound was suggestive of hepatomegaly with well-defined iso-hypoechoic lesion with a peripheral rim of hypodensity in segment IV extending to I, III, V, and VIII measuring $8.2 \times 9.5 \times 8.3 \mathrm{~cm}$ with atypical appearance causing
${ }^{1-7}$ Department of Gastroenterology, Topiwala National Medical College and BYL Nair Hospital, Mumbai, Maharashtra, India

Address reprint requests to: Ravi Thanage, Department of Gastroenterology, Topiwala National Medical College and BYL Nair Hospital, Mumbai, Maharashtra, India, Phone: +91 9028785843, e-mail: ravi.thanage@gmail.com

How to cite this article: Thanage $R$, Jain $S$, Sonthalia $N$, et al. An Enigmatic Liver Mass in a Child. Euroasian J Hepato-Gastroenterol 2019;9(2):104-107.

Source of support: Nil

Conflict of interest: None

Table 1: Laboratory investigations

\begin{tabular}{|c|c|c|}
\hline Laboratory parameters & $\begin{array}{l}\text { Investigations } \\
\text { at other center }\end{array}$ & $\begin{array}{l}\text { Investigations } \\
\text { at our center }\end{array}$ \\
\hline Hemoglobin (13-16 g/dL) & 9.5 & 7.6 \\
\hline $\operatorname{TLC}^{*}(4,000-11,000 / \mu \mathrm{L})$ & 16,000 & 27,000 \\
\hline Platelets $\left(150-450 \times 10^{3} / \mu \mathrm{L}\right)$ & 240 & 210 \\
\hline $\mathrm{AST}^{\dagger}(40 \mathrm{IU} / \mathrm{L})$ & 76 & 66 \\
\hline $\mathrm{ALT}^{\ddagger}(40 \mathrm{IU} / \mathrm{L})$ & 37 & 42 \\
\hline Total bilirubin (0.3-1 mg/dL) & 7.5 & 3.6 \\
\hline Direct bilirubin $(0.1-0.4 \mathrm{mg} / \mathrm{dL})$ & 5 & 2.1 \\
\hline $\operatorname{ALP}^{\S}(21-120 \mathrm{IU} / \mathrm{L})$ & 512 & 473 \\
\hline GGT" (12-48 IU/L) & 163 & 126 \\
\hline Total protein $(6-7.5 \mathrm{~g} / \mathrm{dL})$ & 7.7 & 8.5 \\
\hline Albumin (3-4.5 g/dL) & 2.7 & 2.5 \\
\hline INR $^{\text {I }}$ & 1.1 & 1 \\
\hline
\end{tabular}

${ }^{*}$ Total leukocytes count; ${ }^{\dagger}$ spartate aminotransferase; ${ }^{\ddagger}$ alanine aminotrans-

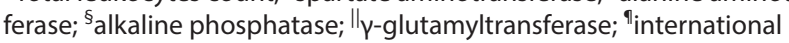
normalised ratio 

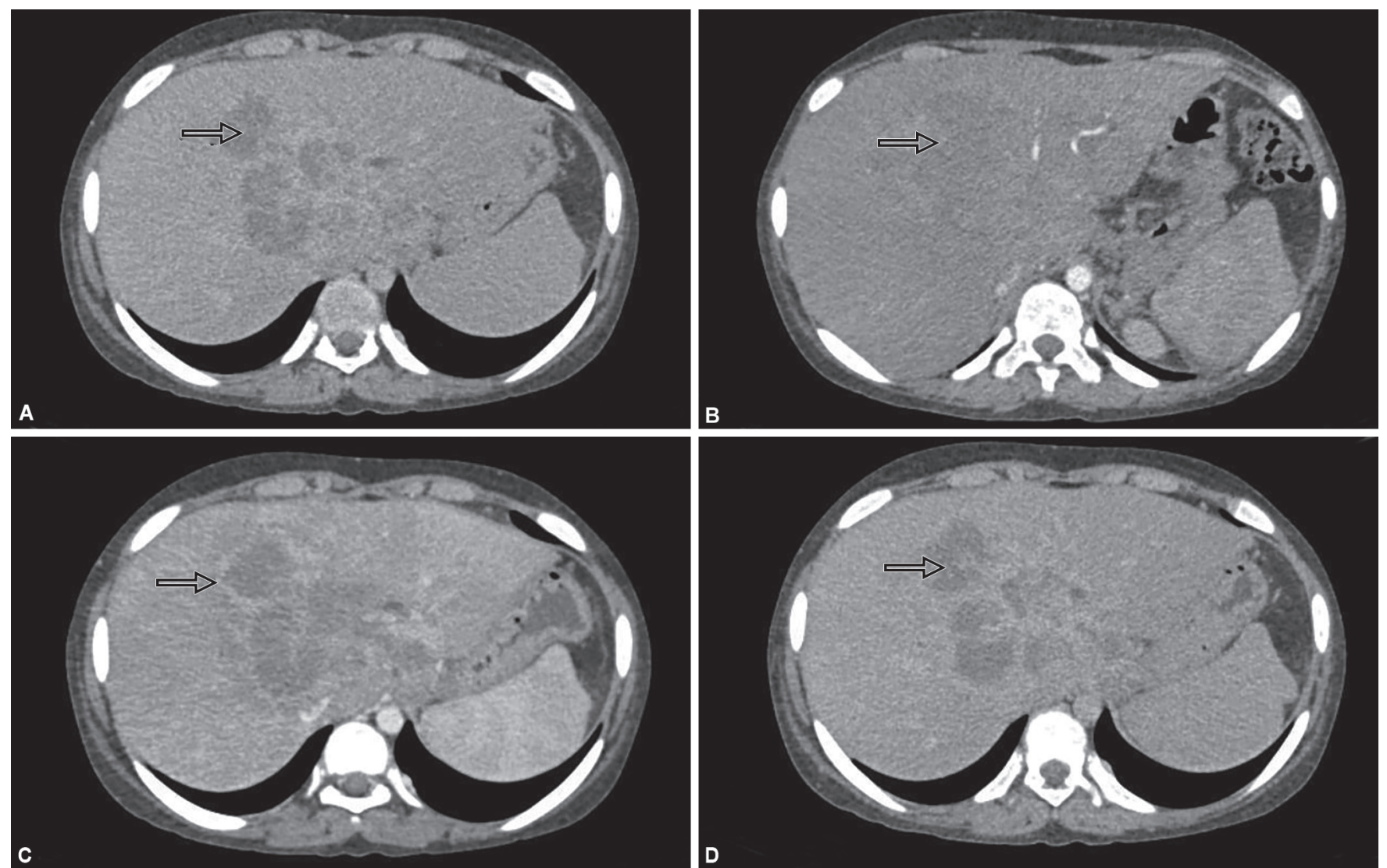

Figs 1 A to D: CT scan of liver showing a large irregular ill-defined heterogeously enhancing mass involving segment IV, V, and VIII: (A) Plain film showing hypodense mass; (B) Contrast enhanced image showing isodense lesion; (C) Venous phase showing heterogenous peripheral enhancement; (D) Delayed phase image showing no enhancement

mild intrahepatic biliary radicles dilatation (IHBRD), portal vein thrombosis with formation of peri-portal and peri-gallbladder collaterals with moderate splenomegaly. Computed tomography of abdomen (Fig. 1) showed liver size $18.5 \mathrm{~cm}$, large irregular ill-defined coalescing heterogenously enhancing lesion $8.6 \times 7.4 \times 6.6 \mathrm{~cm}$ in segment V, VIII, and IVA. The lesion was encasing the left hepatic duct with confluence and resultant IHBRD (left more than right), and also encasing hepatic artery. Portal vein was not visualized in its entire extent and was replaced by multiple collaterals in pericholedochal area and at portahepatis. The spleen size was $14.6 \mathrm{~cm}$.

Histopathologic examination of the hepatic tissue obtained by an ultrasound-guided biopsy demonstrated intrahepatic cholestasis, young fibrosed tissue impregnated with bile and fibrosis associated with resolving inflammation. The patient started on warfarin $3 \mathrm{mg}$ daily for portal vein thrombosis and hepato-safe ATT (tab. levoflox 250 mg, ethambutol 400 mg, inj. streptomycin) empirically.

We reevaluated patient. The results of investigations revealed that his hemoglobin and bilirubin levels had decreased, while leucocytosis, $\gamma$-globulins, alkaline phosphatase, and GGT remained high (Table 1). Ultrasound abdomen demonstrated findings similar to the earlier examination although portal vein was replaced by cavernoma.

Magnetic resonance imaging Gd-BOPTA (a liver specific contrast agent) scan (Fig. 2) showed, T1-hypointense and T2-hyperintense lesions in segment VIII of the right lobe of the liver with extension in porta engulfing common hepatic duct with delayed enhancement possibility of a fibrous tumor.
A repeat liver biopsy was performed. The liver parenchyma showed extensive areas of fibrosis, marked ductular proliferation, bile stasis, and mixed lymphoplasmacytic cell infiltrate with eosinophils (Fig. 3). Intrahepatic cholestasis, focal steatosis, and sinusoidal dilatation with canalicular cholestasis was also noted. Bacterial and tubercular cultures of biopsy sample were negative.

Although serum IgG4 levels were elevated $(7.88 \mathrm{~g} / \mathrm{L}$, reference value: $1.9 \mathrm{~g} / \mathrm{L}$ ), lgG4 staining of liver biopsy was negative, in view of these findings, a diagnosis of IPT was made, ATT was discontinued, and treatment was initiated with oral prednisolone $1 \mathrm{mg} / \mathrm{kg}$ dose.

Ultrasound abdomen done in view of fever on the 20th day after initiation of treatment showed a large $10 \times 10.5 \times 12 \mathrm{~cm}$ loculated predominantly cystic lesion in the right lobe of the liver with internal septation, thick echoes with internal vascularity, and a few small foci of calcification. The patient was treated with intravenous meropenem ( $40 \mathrm{mg} / \mathrm{kg} / \mathrm{day}$, in two divided doses) and vancomycin ( $20 \mathrm{mg} / \mathrm{kg} /$ day in two divided doses) in view of growth of Klebsiella pneumoniae in the pus drained showing sensitivity to these antimicrobial agents. After 5 days of therapy, the draining fluid became purulent and greenish in color. The tests showed the presence of bile salts and bile pigments in the draining fluid, suggesting a bile leak.

The patient underwent endoscopic retrograde cholangiopancreatography and left hepatic duct stenting. The drain output progressively decreased, though the abscess cavity did not collapse completely. The patient was discharged 14 days after the leak. He had two febrile episodes requiring intravenous antibiotic therapy over the past 3 months, clinically, he had no jaundice, had increased appetite 

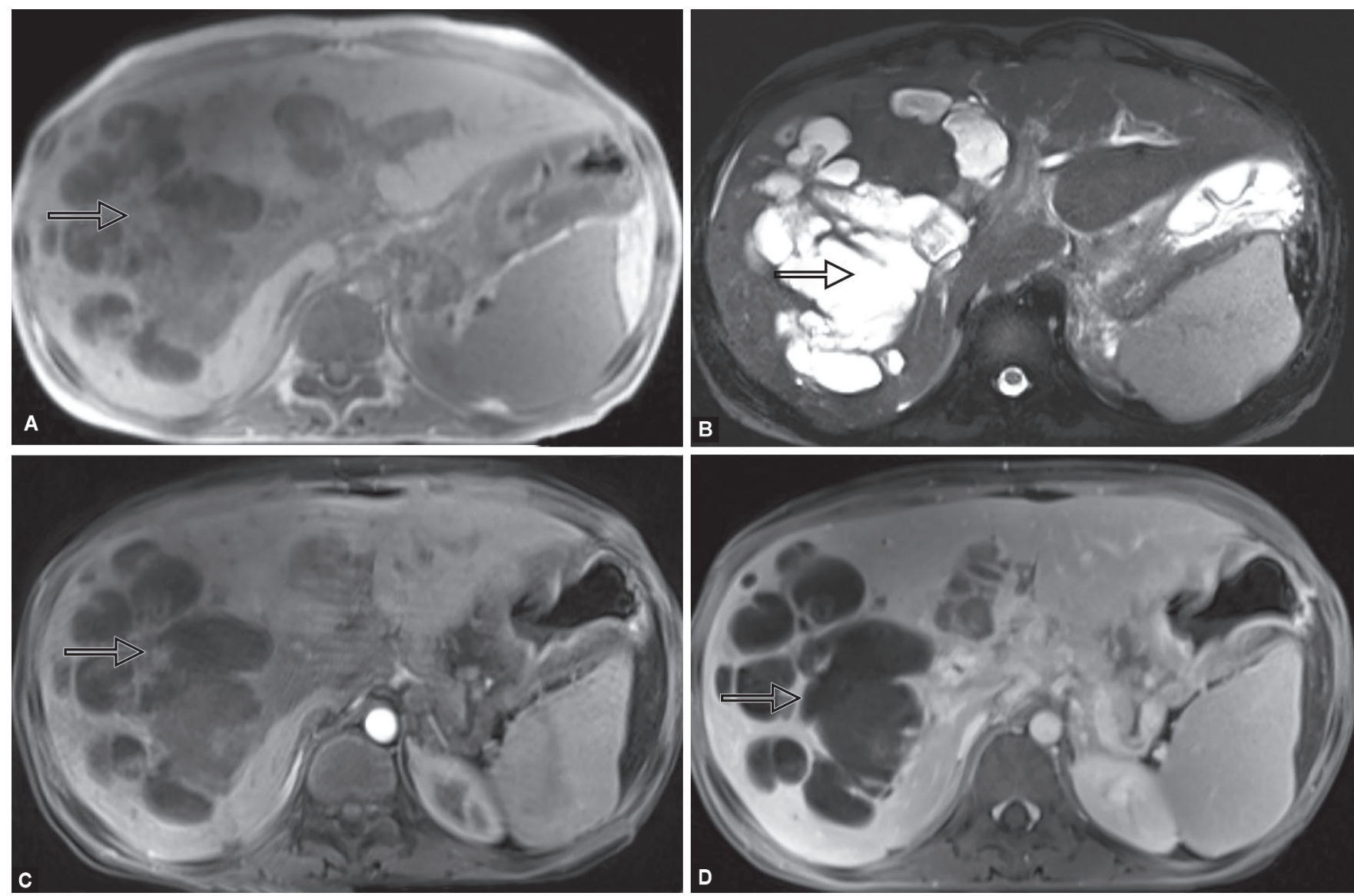

Figs 2A to D: Magnetic resonance imaging of liver showing heterogenous lesion predominantly involving right lobe of liver: (A) T1W image showing hypointense lesion; (B) T2W image showing hyperintense lesion; (C) Postcontrast no enhancement seen; (D) Delayed phase image showing slight enhancement

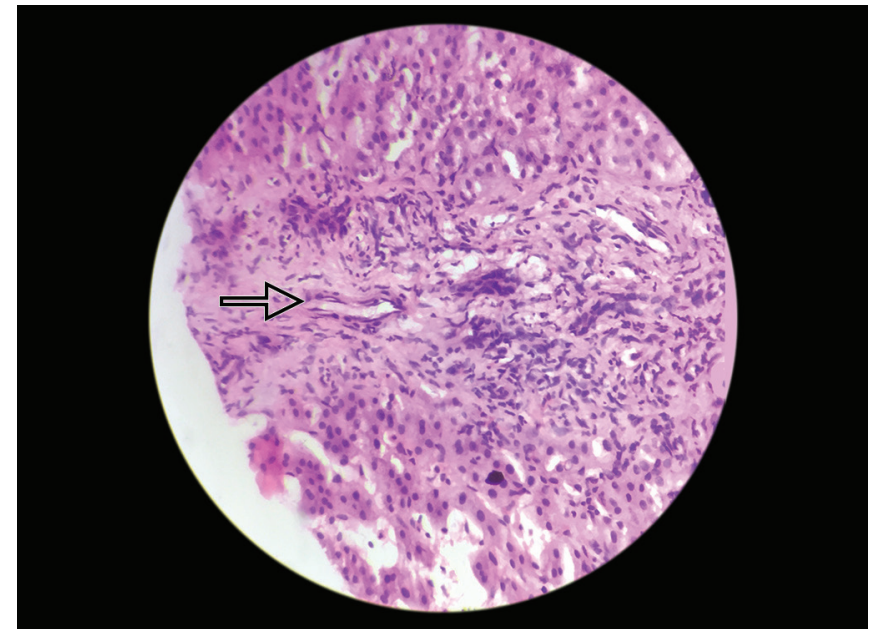

Fig. 3: Liver biopsy showing mixed inflammatory infiltrate with extensive fibrosis (100x magnification)

and weight gain. Patient's solid tumor has now developed into a chronic abscess cavity with a resultant decrease in the size of a cavity.

\section{Discussion}

Inflammatory pseudotumor is a relatively rare disease characterized by chronic infiltration of inflammatory cells and the area of fibrosis. ${ }^{1}$
It is also known as plasma cell granuloma due to the predominance of plasma cells in the lesion. ${ }^{2}$ Inflammatory pseudotumor occurs most commonly in the lung, rarely found in other locations, including eye, spleen, lymph node, and liver. ${ }^{3-5}$ Etiology of hepatic IPT remains uncertain although, infectious condition, an autoimmune phenomenon (IgG4-related diseases), or local inflammatory changes of cholangitis have been suggested as possible etiologies. ${ }^{6-10}$

It is difficult to differentiate IPT of liver from malignant tumors, such as atypical hepatocellular carcinoma, intrahepatic cholangiocarcinoma, and metastatic cancer ${ }^{11,12}$ or liver abscess showing incomplete liquefaction and granulation. ${ }^{13,14}$

Age of presentation can range from 3 months to 15 years with an almost equal male-to-female ratio. Fever $40 \%$, abdominal pain $43 \%$, and abdominal mass $28 \%$ are major symptoms, constitutional symptoms, such as anorexia, weight loss, and night sweats were seen in one-fifth of patients. Jaundice at presentation is caused by the presence of tumor in the porta hepatis. Tumor would be a single lesion almost $>90 \%$ of time with size ranging from $2 \mathrm{~cm}$ to $14 \mathrm{~cm}$. a-Fetoprotein level would be normal. ${ }^{15,16}$

The imaging findings of IPT of the liver are nonspecific. The lesion can manifest itself as a single focal or multifocal mass. These lesions appear as hypoechoic or hyperechoic masses with increase through transmission and septation on ultrasound imaging. Contrast-enhanced computed tomography imaging shows various patterns of enhancement, including heterogeneous enhancement, homogeneous enhancement of septa, peripheral 
enhancement with delayed central filling, and no enhancement or central necrosis. On magnetic resonance images, these lesions are usually $\mathrm{T} 1$ hypointense and $\mathrm{T} 2$ hyperintense with heterogeneous enhancement. $^{17}$

There are two major subtypes based on histological features, namely, lymphoplasmacytic and fibrohistiocytic types. The lymphoplasmacytic type is defined by an inflammatory process, mainly involving lymphoplasmacytic infiltration (IgG4 positive plasma cells), obliterative phlebitis and cholangitis with periductal fibrosis are common features. Lesions are usually found around the hepatic hilum. The fibrohistiocytic type was defined as having abundant histiocytic infiltration involving foamy cells or multinucleated giant cells. Venous occlusion with scanty inflammation and cholangitis without periductal fibrosis were often seen. Mostly occurred in the peripheral hepatic parenchyma as mass-forming lesions. ${ }^{8}$

Until recently, surgical resection was the preferred treatment for pseudotumors. With the discovery of IgG4-related disease, use of corticosteroids in pseudotumor has increased..$^{18}$ Many case reports have suggested variable success with corticosteroid use. Shibata et al. ${ }^{19}$ had reported a similar case of 72 -year-old man with hepatic IPT developed abscess after starting corticosteroids therapy, abscess resolved in the next 6 months. In this patient, steroid had resulted in liver abscess with recurrent hospital admission for fever.

Take home message is hepatic IPT is a rare benign tumor with vague symptoms of abdominal pain, low-grade fever, and loss of appetite. They are difficult to differentiate from malignant tumors as their variable imaging findings. To avoid inadvertent surgery, histologic confirmation of the hepatic mass is essential. Whenever in doubt after imaging, a biopsy will help in the diagnosis of a rare differential diagnosis, such as hepatic IPT. Antitubercular therapy should not be used without diagnosis. Steroids should be used with caution with close follow-up to prevent iatrogenic complications like a chronic liver abscess. Surgical treatment should be offered after failed medical management.

\section{References}

1. Torzilli G, Inoue K, Midorikawa Y, et al. Inflammatory pseudotumors of the liver: prevalence and clinical impact in surgical patients. Hepatogastroenterology 2001;48(40):1118-1123.

2. Horiuchi R, Uchida T, Kojima T, et al. Inflammatory pseudotumor of the liver. Clinicopathologic study and review of the literature. Cancer 1990;65(7):1583-1590. DOI: 10.1002/1097-0142 (19900401)65:7<1583::AID-CNCR2820650722>3.0.CO;2-L.

3. Coffin CM, Humphrey PA, Dehner LP. Extrapulmonary inflammatory myofibroblastictumor: a clinical and pathological survey. Semin Diagn Pathol 1998;15(2):85-101.

4. Melloni G, Carretta A, Ciriaco P, et al. Inflammatory pseudotumor of the lung in adults. Ann Thorac Surg 2005;79(2):426-432. DOI: 10.1016/j.athoracsur.2004.07.077.
5. Kawaguchi T, Mochizuki K, Kizu T, et al. Inflammatory pseudotumor of the liver and spleen diagnosed by percutaneous needle biopsy. World J Gastroenterol 2012;18(1):90-95. DOI: 10.3748/wjg.v18. i1.90.

6. White JE, Chase CW, Kelley JE, et al. Inflammatory pseudotumor of the liver associated with extrahepatic infection. South Med J 1997;90(1):23-29. DOI: 10.1097/00007611-199701000-00005.

7. Yamamoto $H$, Yamaguchi $H$, Aishima $S$, et al. Inflammatory myofibroblastictumor versus IgG4-related sclerosing disease and inflammatory pseudotumor: a comparative clinicopathologic study. Am J Surg Pathol 2009;33(9):1330-1340. DOI: 10.1097/ PAS.0b013e3181a5a207.

8. Zen Y, Fujii T, Sato Y, et al. Pathological classification of hepatic inflammatory pseudotumor with respect to IgG4-related disease. Mod Pathol 2007;20(8):884-894. DOI: 10.1038/modpathol.3800836.

9. Nakanuma Y, Tsuneyama K, Masuda S, et al. Hepatic inflammatory pseudotumor associated with chronic cholangitis: report of three cases. Hum Pathol 1994;25(1):86-91. DOI: 10.1016/00468177(94)90176-7.

10. Yoon $\mathrm{KH}, \mathrm{Ha} \mathrm{HK}$, Lee JS, et al. Inflammatory pseudotumor of the liver in patients with recurrent pyogenic cholangitis: CT-histopathologic correlation. Radiology 1999;211(2):373-379. DOI: 10.1148/radiology. 211.2.r99ma36373.

11. Kitajima K, Shiba H, Nojiri T, et al. Intrahepatic cholangiocarcinoma mimicking hepatic inflammatory pseudotumor. J Gastrointest Surg 2007;11(3):398-402. DOI: 10.1007/s11605-006-0071-1.

12. Ishida $H$, Tatsuta $M$, Furukawa $H$, et al. Multiple inflammatory pseudotumors mimicking liver metastasis from colon cancer: report of a case. Surg Today 2000;30(6):530-533. DOI: 10.1007/ s005950070121.

13. Fukuya $\mathrm{T}$, Honda $\mathrm{H}$, Matsumata $\mathrm{T}$, et al. Diagnosis of inflammatory pseudotumor of the liver: value of CT. AJR Am J Roentgenol 1994;163(5):1087-1091. DOI: 10.2214/ajr.163.5.7976880.

14. Pack GT, Baker HW. Total right hepatectomy; report of a case. Ann Surg 1953;138(2):253-258. DOI: 10.1097/00000658-195308000-00012.

15. Nagarajan S, Jayabose S, McBride W, et al. Inflammatory myofibroblastictumor of the liver in children.J Pediatr Gastroenterol Nutr 2013;57(3):277-280. DOI: 10.1097/MPG.0b013e31829e0b3b.

16. Al-Hussaini $\mathrm{H}$, Azouz $\mathrm{H}, \mathrm{Abu}$-Zaid A. Hepatic inflammatory pseudotumor presenting in an 8-year-old boy: a case report and review of literature. World J Gastroenterol 2015;21(28):8730-8738. DOI: $10.3748 /$ wjg.v21.i28.8730.

17. Patnana $M$, Sevrukov $A B$, Elsayes $K M$, et al. Inflammatory pseudotumor: the great mimicker. AJR Am J Roentgenol 2012;198(3):217-227. DOI: 10.2214/AJR.11.7288.

18. Zen Y, Harada K, Sasaki M, et al. IgG4-related sclerosing cholangitis with and without hepatic inflammatory pseudotumor, and sclerosing pancreatitis-associated sclerosing cholangitis: do they belong to a spectrum of sclerosing pancreatitis? Am J Surg Pathol 2004;28(9):1193-1203. DOI: 10.1097/01.pas.0000136449.37936.6c.

19. Shibata M, Matsubayashi $H$, Aramaki T, et al. A case of IgG4-related hepatic inflammatory pseudotumor replaced by an abscess after steroid treatment. BMC Gastroenterol 2016;16(1):89. DOI: 10.1186/ s12876-016-0504-6. 